ARTICLE

\title{
The Set1 N-terminal domain and Swd2 interact with RNA polymerase II CTD to recruit COMPASS
}

Hyun Jin Bae ${ }^{1,6}$, Marion Dubarry ${ }^{2,6}$, Jongcheol Jeon (10 1,3, Luis M. Soares ${ }^{1,5}$, Catherine Dargemont ${ }^{4}$, Jaehoon Kim (iD ${ }^{3}$, Vincent Geli (iD ${ }^{2 \otimes} \&$ Stephen Buratowski (iD) ${ }^{1 凶}$

Methylation of histone $\mathrm{H} 3$ lysine 4 (H3K4) by Set1/COMPASS occurs co-transcriptionally, and is important for gene regulation. Set1/COMPASS associates with the RNA polymerase II C-terminal domain (CTD) to establish proper levels and distribution of H3K4 methylations. However, details of CTD association remain unclear. Here we report that the Set1 $\mathrm{N}$-terminal region and the COMPASS subunit Swd2, which interact with each other, are both needed for efficient CTD binding in Saccharomyces cerevisiae. Moreover, a single point mutation in Swd2 that affects its interaction with Set1 also impairs COMPASS recruitment to chromatin and H3K4 methylation. A CTD interaction domain (CID) from the protein Nrd1 can partially substitute for the Set1 $\mathrm{N}$-terminal region to restore CTD interactions and histone methylation. However, even when Set1/COMPASS is recruited via the Nrd1 CID, histone H2B ubiquitylation is still required for efficient H3K4 methylation, indicating that $\mathrm{H} 2 \mathrm{Bub}$ acts after the initial recruitment of COMPASS to chromatin.

\footnotetext{
${ }^{1}$ Department of Biological Chemistry and Molecular Pharmacology, Harvard Medical School, Boston, MA 02115, USA. ${ }^{2}$ Cancer Research Center of Marseille (CRCM), U1068 Inserm, UMR7258 CNRS, Aix Marseille University (AMU), Institut Paoli-Calmettes. Equipe labellisée Ligue contre le cancer, Marseille 13009, France. ${ }^{3}$ Department of Biological Sciences, Korea Advanced Institute of Science and Technology, Daejeon 34141 , South Korea. ${ }^{4}$ Institute of Human Genetics, UMR 9002 CNRS, Montpellier 34396, France. ${ }^{5}$ Present address: Foghorn Therapeutics, Cambridge, MA 02142, USA. ${ }^{6}$ These authors contributed

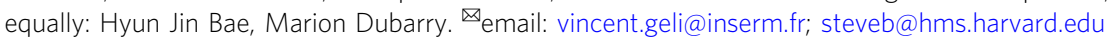


E ukaryotic gene transcription is regulated by posttranslational modifications of histone tails, which include phosphorylation, acetylation, ubiquitylation, and methylation ${ }^{1}$. Specific methylations of histone lysine residues correlate with transcription activity or repression. For instance, methylations on $\mathrm{H} 3 \mathrm{~K} 4, \mathrm{~K} 36$, and $\mathrm{K} 79$ are enriched over genes actively transcribed by RNA polymerase II (RNApII), whereas $\mathrm{H} 3 \mathrm{~K} 9$ and $\mathrm{H} 3 \mathrm{~K} 27$ methylations are highest in transcriptionally inactive regions ${ }^{2}$. Methylation of H3K4 (H3K4me) has aroused particular interest ${ }^{3}$. In mammals, this mark can be deposited by multiple complexes (Setd1a, Setd1b, Mll1, Mll2, Mll3, and Mll4), all of which share a module comprising WDR5, RbBP5, ASH2L, and DPY-30 (called WRAD) that associates with the catalytic SET domain 4 . Each complex is endowed with additional proteins that determine their recruitment and biological functions ${ }^{5}$.

In budding yeast, all H3K4 methylation is catalyzed by a single Set 1 complex (called Set1C or COMPASS) in which Set1 acts as a scaffold for seven subunits (Bre2, Sdc1, Shg1, Spp1, Swd1, Swd2, and $S w d 3)^{6-9}$. Of these, $S w d 2$ is the only subunit that is essential for viability. However, this requirement stems from its additional role as a component of the RNA $3^{\prime}$ end processing and termination complex, APT (Associated with Pta1) ${ }^{10,11}$. It is unclear whether Swd2 plays the same role in both APT and COMPASS. Protein interaction studies have shown that the Set1 SET domain associates with the WRAD homologs Swd1, Swd3, Bre2, and Sdc1, the N-SET domain associates with Spp1, the Set1 central region binds Shg1, and the Set1 N-terminal region contacts Swd27,12-15. Swd1 also shows some interactions with the Set1 Nterminal region ${ }^{14,16}$. This organization of COMPASS structure was confirmed by cryo-electron microscopy ${ }^{17-19}$.

Control of COMPASS activity involves a complex set of interactions. Remarkably, higher level H3K4 methylation by Set1 requires prior ubiquitylation on histone $\mathrm{H} 2 \mathrm{~B}(\mathrm{H} 2 \mathrm{Bub})^{20,21}$. Spp1 contact with Swd1/Swd3 is crucial for H2Bub-dependent H3K4 methylation $^{16}$. Deletion or depletion of individual COMPASS subunits differentially impairs Set1 stability and the pattern of H3K4 methylation along active genes ${ }^{12,14,22-24}$. Particularly relevant to this study, depletion of the WD40 repeat protein Swd2 strongly destabilizes Set1 and reduces H3K4 methylation 7,10,25,26. Set1 activity is positively regulated by the Set1 double RNA recognition motif (dRRM), but inhibited by a centrally located auto-inhibitory domain ${ }^{27,28}$. Set1 dRMM binding to nascent RNA may affect COMPASS distribution along transcription units and subsequent deposition of the H3K4me3 mark ${ }^{29}$. Surprisingly, combined deletion of the N-terminal, dRMM, and central domains leads to overexpression of a truncated Set1 protein with mistargeted H3K4 methylation ${ }^{16,30,31}$. Finally, mutations in $\mathrm{H} 3 \mathrm{~K} 4$ lead to Set1 degradation, indicating a feedback mechanism to control enzyme levels ${ }^{30}$.

The C-terminal domain (CTD) of Rpb1, the largest subunit of RNApII, consists of multiple Tyr1-Ser2-Pro3-Thr4-Ser5-Pro6Ser7 (YSPTSPS) heptad repeats ${ }^{32}$. Chromatin immunoprecipitation (ChIP) with antibodies against individual phosphorylated CTD residues shows that phosphorylation of Serine 5 (Ser5P) peaks near the promoter, whereas Serine 2 phosphorylation (Ser2P) increases later during elongation ${ }^{33}$. Set1C/COMPASS cotranscriptionally associates with Ser5-phosphorylated RNApII ${ }^{34}$ to produce a gradient of $\mathrm{H} 3 \mathrm{~K} 4$ methylation that begins at the +1 nucleosome and tails off with distance from the promoter ${ }^{1,2}$. This $5^{\prime}$ targeting is compounded over multiple transcription cycles ${ }^{35}$, leading to the canonical peak of $\mathrm{H} 3 \mathrm{~K} 4 \mathrm{me} 3$ near the promoter, followed by $\mathrm{H} 3 \mathrm{~K} 4$ dimethylation $(\mathrm{H} 3 \mathrm{~K} 4 \mathrm{me} 2)$ and monomethylation (H3K4me1) further downstream.

Binding of COMPASS to the RNApII CTD has been proposed to rely on the Paf1 complex ${ }^{36,37}$. However, direct interactions of COMPASS with either Paf1 complex or RNApII have yet to be characterized. The recruitment of COMPASS to transcribed genes was also proposed to rely on interaction between $\mathrm{Swd} 2$ and $\mathrm{H} 2 \mathrm{Bub}^{23}$. However, this model is not supported by in vitro reconstitution experiments showing that COMPASS lacking Swd2 still methylates $\mathrm{H} 3 \mathrm{~K} 4$ in an $\mathrm{H} 2 \mathrm{~B}$ ubiquitylation-dependent manner ${ }^{14}$. Therefore, it remains unclear how COMPASS interacts with RNApII, and whether other proteins participate in the interaction.

Here, we provide biochemical and yeast two-hybrid evidence showing that the Set1 N-terminal region and Swd2 together mediate interaction with the Rpb1 CTD. Deletion of the first 200 amino acids of Set1 results in both loss of RNApII CTD binding and reduction of $\mathrm{H} 3 \mathrm{~K} 4 \mathrm{me} 2$ and $\mathrm{H} 3 \mathrm{~K} 4 \mathrm{me} 3$ levels. These effects can be partially reversed by replacing this region of Setl with the Nrd1 CTD-interacting domain (CID), which specifically binds Ser5P CTD ${ }^{38}$. The role of Swd2 in COMPASS recruitment is further supported by the effects of a point mutation in $\mathrm{Swd} 2$ that compromises its interaction with Set1, the recruitment of the complex to chromatin, and H3K4 methylation. Finally, when COMPASS is recruited by the Nrd1 "bypass" mechanism, the Paf1 complex and H2Bub are still required for H3K4 methylation, suggesting that $\mathrm{H} 2 \mathrm{Bub}$ acts downstream of initial COMPASS recruitment to elongation complexes.

\section{Results}

The Set1 N-terminal domain mediates RNApII association. Our previous analysis of Set $1 \mathrm{~N}$-terminal truncations found that deletion of the first 200 amino acids of Set 1 strongly reduced both $\mathrm{H} 3 \mathrm{~K} 4 \mathrm{me} 2$ and $\mathrm{me}^{30}$. This region of Set1 interacts with the Swd2 subunit of COMPASS ${ }^{14}$. Interestingly, the mammalian Swd2 homolog Wdr82 preferentially binds Ser5P-CTD in vitro, and has thus been proposed to mediate Setd1A complex recruitment to elongating $\mathrm{RNApII}^{15}$.

To test if the drop in H3K4me levels upon Set1 N-terminal truncation correlates with reduced association of COMPASS with Ser5P-CTD, a strain lacking the SET1 gene (set1D) was transformed with plasmids expressing either full-length Set1 or a truncation lacking 100 or $200 \mathrm{~N}$-terminal amino acids (S $\Delta 100$ and $S \Delta 200$, Fig. 1a). Consistent with our previous results ${ }^{30}$, Set1 and $\mathrm{S} \Delta 100$ supported $\mathrm{H} 3 \mathrm{~K} 4 \mathrm{me} 3$ and $\mathrm{H} 3 \mathrm{~K} 4 \mathrm{me} 2$, but $\mathrm{S} \Delta 200$ did not (Fig. 1b). To monitor binding to Ser5P-CTD, the epitopetagged Set1 proteins were immunoprecipitated using anti-FLAG conjugated beads ( $\alpha$-FLAG), followed by immunoblotting for Ser5P-CTD. As shown in Fig. 1c, N-terminal deletion of Set1 $(\mathrm{S} \triangle 200)$ attenuated Set1 association with Ser5P-CTD and total RNApII. This result suggests that the N-terminal domain of Set1 (aa 1-200) that interacts with Swd2 is also important for the direct or indirect association of Set1 with RNApII.

The N-terminal region of Set1 interacts with the Rpb1 CTD. Evidence for a direct interaction between COMPASS and the CTD of RNApII subunit Rpb1 has been lacking. A genome-wide yeast two-hybrid $(\mathrm{Y} 2 \mathrm{H})$ screen was performed using full length Set1 fused to the Gal4 DNA binding domain (BD) as bait. Remarkably, a 14-CTD repeat fragment fused to the Gal4 activation domain $(\mathrm{AD})$ was isolated as a robust interactor. The 14CTD repeat construct was then tested with each of the other COMPASS subunits, but Set1 was the only subunit that showed a positive $\mathrm{Y} 2 \mathrm{H}$ interaction (Supplementary Fig. 1a). To further map the CTD interaction, Set1 was divided into five fragments encompassing previously reported functional domains (Fig. 2a) ${ }^{14}$. Immunoblotting with antibodies against Gal4 BD and Set1 confirmed fusion protein expression (Supplementary Fig. 1b, c). Y2H analysis showed that full-length Set1, the F1(aa 1-236), and Set1 (1-200) fusions activated the Gal4-driven HIS3 and ADE2 
a

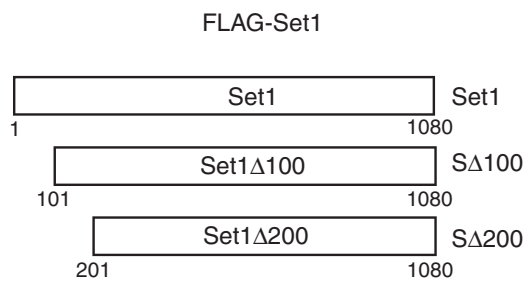

b

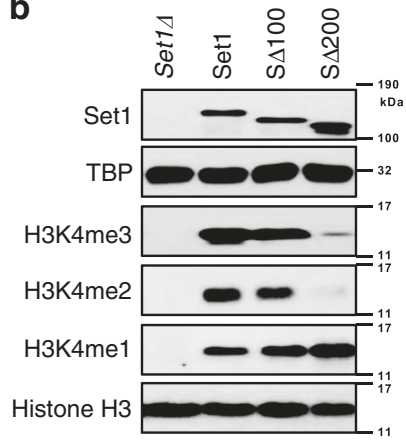

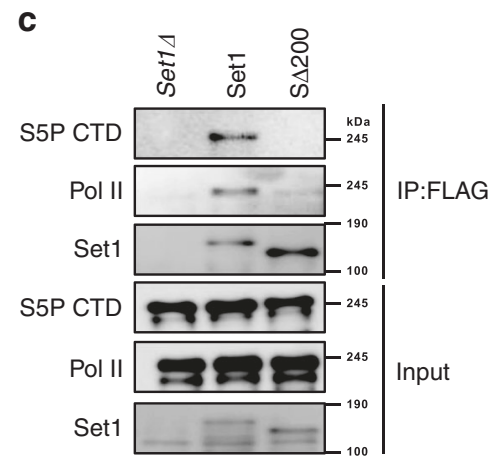

Fig. 1 The Set1 $\mathbf{N}$-terminal domain is needed for RNApll interaction and proper H3K4 methylation. a Schematic of Set1 constructs used, with numbers below diagram indicating the amino acid residues from the wild-type proteins. A FLAG-tag at the N-terminus of all constructs is not shown. $\mathbf{b}$ Full length Set1 (Set1) and N-terminal truncated mutants $(S \Delta 100, S \Delta 200)$ were transformed into set1 $\Delta$ cells. Whole cell lysates were separated by SDS-PAGE and analyzed by immunoblotting using the indicated antibodies. Lysates from cells transformed with empty vector plasmid (set1 1 ) served as a negative control. TATA-binding protein (TBP) and histone H3 were used as loading controls. c FLAG-tagged full-length (Set1) or Set1 $\Delta 200$ (S $\Delta 200$ ) were expressed in set14 cells. Set1 proteins were immunoprecipitated with anti-FLAG beads (IP:FLAG) from whole cell lysates and analyzed by immunoblotting using antibodies for Ser5P (S5P CTD: 3E8) or total Rpb1 (POL II: 8WG16). Immunoprecipitations from cells transformed with empty vector plasmid (set14) served as a negative control. Bottom panels show input samples, and upper panels show proteins bound to FLAG beads after precipitation. Source data are provided as a Source data file.

$\mathbf{a}_{1}$

\begin{tabular}{|c|c|c|c|c|c|}
\hline & & & 79 & \multicolumn{2}{|c|}{938} \\
\hline & RRM1 & RRM2 & & n-SET & SET \\
\hline F1 & & & & & \\
\hline $1-236$ & & & $\frac{r}{590}$ & $\mathrm{~F} 4$ & F5 \\
\hline
\end{tabular}

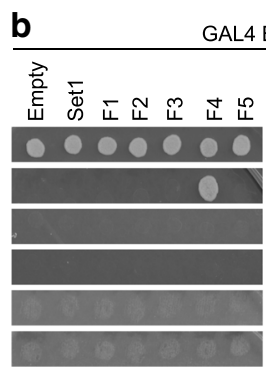

GAL4 AD
GAL4 BD-Set1

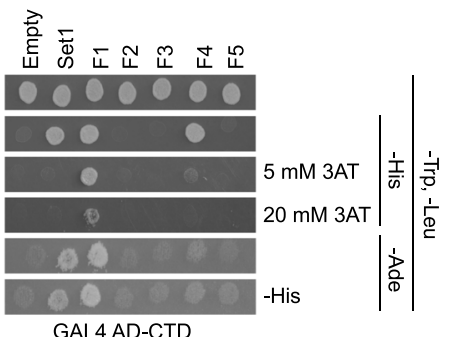

C

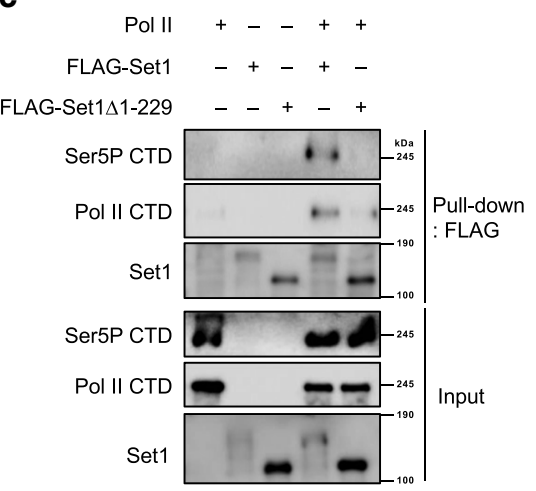

Fig. 2 The Set1 N-terminal region binds the Rpb1 CTD. a Schematic of Set1 fragments fused to GAL4 BD for yeast two hybrid assay. b GAL4 BD-Set1 fusions were co-expressed with GAL4 AD-CTD in Y2H strain PJ69-4A, which has both HIS3 and ADE2 reporters for Gal4 activation. Plasmid expressing only the GAL4 AD was used as a negative control. Cells were grown for 2 days on synthetic complete (SC) media plates lacking the indicated amino acids. Five or $20 \mathrm{mM}$ of 3-aminotriazol (3AT) was added to media as indicated to increase HIS3 selection stringency. Cells were also replica plated to -Ade or -Ade, -His plates to monitor the ADE2 reporter. c COMPASS containing FLAG-tagged Set1 or a derivative lacking residues 1-229 was incubated with purified RNApll. Bottom panels show input samples, and upper panels show proteins bound to FLAG beads after precipitation. Source data are provided as a Source data file.

reporters, signifying interaction with RNApII CTD (Fig. 2b, Supplementary Fig. 1d). The weaker signal with the full-length Gal4 BD-Set1 fusion is due to reduced expression (Supplementary Fig. 1b, c), consistent with earlier findings that wild-type Set1 stability is tightly regulated to keep protein levels low ${ }^{30}$. The F4 (aa 770-945) fragment, which includes the Spp1 interacting region, also weakly activated the HIS3 reporter, but independently of the Gal4 AD-CTD construct. Adding 3-aminotriazol (3AT) to the media selects for higher levels or HIS3 expression, where only the F1 fragment scored as positive (Fig. 2b).

To test whether the interactions were direct, baculovirusexpressed COMPASS ${ }^{16}$ was incubated with RNApII purified from yeast (generous gift from Dr. Naruhiko Adachi) and immunoprecipitated via the FLAG epitope on Set1. While RNApII readily bound to COMPASS containing full-length Set1, little or no interaction was seen with Set1 $\Delta 1-229$ (Fig. 2c).
Therefore, we conclude that the Set1 N-terminal region is required for RNApII binding.

Swd2 contributes to interaction between COMPASS and RNApII CTD. Wdr82, the mammalian homolog of Swd2, can directly bind Ser5P-CTD in vitro, but also binds the N-terminal region of mammalian Setd $1 \mathrm{~A}^{15}$. We therefore considered the possibility that the amino terminal domain of Set1 interacts with the CTD only indirectly via Swd2. However, an Swd2-CTD interaction was not seen by $\mathrm{Y} 2 \mathrm{H}$ (Supplementary Fig. 1a), arguing against this model. Furthermore, when Swd2 was directly fused to $\mathrm{S} \triangle 200$ and introduced into cells, the fusion protein $(\mathrm{SS} \triangle 200)$ did not restore H3K4 methylation (Supplementary Fig. 2a).

One possible explanation for these results is that CTD actually binds the combination of Swd2 and Set1, either as a composite binding surface, or because one component is required to trigger 


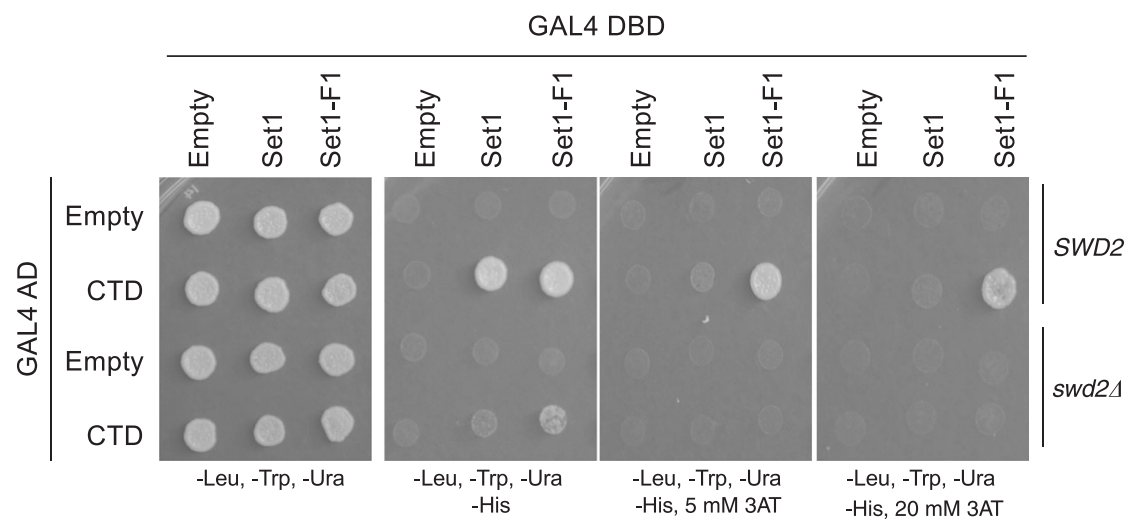

Fig. 3 Swd2 enhances the Set1-CTD Y2H interaction. Gal4 BD fused to full length Set1 (Set1) or amino acids 1-236 (Set1-F1, see Fig. 2) were expressed in $\mathrm{Y} 2 \mathrm{H}$ strain PJ69-4A carrying wild-type (SWD2) or deletion (swd24) alleles of SWD2. They were combined with a Gal4 AD fusion to the Rpb1 CTD (CTD) or the parent vector (Empty). Cells were spotted on the indicated selective plates. Source data are provided as a Source data file.

a CTD-binding conformation in the other. To test this possibility, the $\mathrm{Y} 2 \mathrm{H}$ interaction between the Gal4 AD-CTD fusion and Gal4 BD fused to full-length Set1 or segment F1 was compared in isogenic reporter strains that were either wild-type SWD2 or $s w d 2 \Delta$. The lethality of $s w d 2 \Delta$ was suppressed, as previously described, by expression of a fragment from the termination factor Sen 1 (amino acids 1890-2092, annotated as Sen1(202) ${ }^{25}$ ). The control SWD2 strain was also transformed with the same Sen 1 construct to maintain isogeneity. Immunoblotting once again showed that Gal4 fused to Set1 F1 is expressed at higher levels than the full-length Set1 fusion, but also that $s w d 2 \Delta$ does not affect amounts of either protein (Supplementary Fig. 2b). In the $\mathrm{Y} 2 \mathrm{H}$ assay, activation of the Gal4-responsive HIS3 reporter was severely reduced, but not abolished by $s w d 2 \Delta$ (Fig. 3). In agreement, $s w d 2 \Delta$ strongly reduces $\mathrm{H} 3 \mathrm{~K} 4 \mathrm{me} 3$ in cells with wildtype Set1, but not in cells with the Set1 N-terminal truncation (S $\Delta 200)$ (Supplementary Fig. 2c). These results suggest that Swd2, while not absolutely required, strongly promotes proper interaction between the Set1 and CTD fusion proteins.

Additional deletions more precisely mapped Swd2 binding to aa $124-229$ of Set $1^{16}$. In agreement with this finding, deleting amino acids 200-210 from the Set1-F1 fragment (F1 $\left.{ }^{\Delta 200-210}\right)$ weakened its yeast two-hybrid interaction with both Swd2 and CTD Gal4 AD fusions (Supplementary Fig. 3a). In the context of full length Set1, $\Delta 200-210$ only slightly affected Set1 protein levels, but H3K4me3 levels (Supplementary Fig. 3b) and ChIP of Set 1 to the $5^{\prime}$ region of PMA1 gene were reduced (Supplementary Fig. 3c). These results further show that $\mathrm{Swd} 2$ contributes to the COMPASS-RNApII interaction.

The Nrd1 CID can substitute for the Set1 N-terminal domain. If the primary role of the Set $1 \mathrm{~N}$-terminal region and associated Swd2 subunit is to recruit COMPASS to the elongation complex via CTD binding, it should be possible to replace this domain with another Ser5P-binding protein. The CID from Nrd1 targets the snoRNA termination machinery to $5^{\prime}$ ends by direct binding to Ser5P-CTD ${ }^{38}$. Accordingly, we created a fusion where the CID replaces the N-terminal 200aa of Set1 (Fig. 4a). Strikingly, the Nrd1(CID)-S $\Delta 200$ fusion (NS $\Delta 200)$ partially rescued both bulk H3K4me2 and me3 (Fig. 4b), as well as the ability to coprecipitate Ser5-phosphorylated RNApII (Fig. 4c). To prove that restoration of $\mathrm{H} 3 \mathrm{~K} 4$ methylation patterns by the Nrd1 CID fusion is mediated by CTD binding, we created two Set1 fusions with Nrd1 CID point mutants (D70R or I130R) known to disrupt the $\mathrm{CTD}_{\text {interaction }}{ }^{38}$. Co-immunoprecipitation experiments confirmed that D70RS $\Delta 200$ and I130RS $\Delta 200$ fusion proteins were expressed normally, but had lost the ability to stably bind RNApII
(Fig. 4c). Neither mutant restored $\mathrm{H} 3 \mathrm{~K} 4$ methylation relative to $\mathrm{S} \triangle 200$, as expected if CTD binding is essential for proper COMPASS targeting (Fig. 4b).

To determine if this CID-mediated rescue of $\mathrm{H} 3 \mathrm{~K} 4$ methylation reflects normal or aberrant distribution along genes, H3K4me2 and me3 patterns were analyzed genome-wide by ChIP and high-throughput sequencing (ChIP-Seq). Spiked-in $S$. pombe chromatin was used as an internal control. Both individual gene heat maps (Fig. 4d, e, left and middle panels) and averaged "meta-gene" anchor plots (Supplementary Fig. 4a, b) show that $\mathrm{S} \Delta 200$ significantly reduced $\mathrm{H} 3 \mathrm{~K} 4 \mathrm{me} 3$ peaks. These effects can also be seen in representative Mochiview ${ }^{39}$ genome browser tracks for individual genes (Supplementary Fig. 4c, d), as well as in heat maps quantitating the differences between $S \Delta 200$ and Set 1 FL (Supplementary Fig. 4e, f, left panels). Relative to $\mathrm{S} \Delta 200$, the NS $\Delta 200$ fusion increased both promoter-proximal H3K4me3 and downstream H3K4me2 (Fig. 4d, Supplementary Fig. 4). Thus, the NS $\Delta 200$ methylation patterns are intermediate between wildtype Set1 and $S \Delta 200$ cells, consistent with improved recruitment of COMPASS by the Nrd1 CID. We have previously shown that trimethylation occurs over multiple rounds of transcription ${ }^{35}$, so in mutants with reduced COMPASS occupancy or activity, promoter-proximal nucleosomes maximally attain $\mathrm{H} 3 \mathrm{~K} 4 \mathrm{me}$, while downstream nucleosomes that would normally have H3K4me2 only reach $\mathrm{H} 3 \mathrm{~K} 4 \mathrm{me} 1$, making it appear that the H3K4 methylation gradient has shifted upstream ${ }^{35}$.

Because the NS $\Delta 200$ fusion replaces the Set1 Swd2-binding region with the Nrd1 CID, its interaction with RNApII is predicted to be independent of Swd2. To test this, we compared the behaviors of wild-type Set1, S $\Delta 200$, and NS $\triangle 200$ in SWD2/ set $1 \Delta$ versus $s w d 2 \Delta /$ set $1 \Delta$ cells. As previously seen ${ }^{25,30}$, full-length Set 1 is degraded in cells lacking Swd2. In contrast, levels of S $\Delta 200$ or NS $\Delta 200$ were not reduced in $s w d 2 \Delta$ cells (Fig. $4 \mathrm{f}$ ). H3K4me3, assayed either in bulk (Fig. 4f) or by ChIP (Supplementary Fig. 2c), was strongly stimulated by Swd 2 in SET1 cells, but not in S $\triangle 200$ or NS $\triangle 200$. Deletion of SWD2 also strongly diminished interaction between RNApII and wild-type Set1. In contrast, coimmunoprecipitation of RNApII with NS $\Delta 200$, while lower than wild-type Set1, was independent of Swd2 (Fig. 4g). These observations further suggest that Swd2 and the Set1 N-terminal region cooperate in COMPASS targeting.

The Swd2 WD40 domain is important for COMPASS recruitment. To characterize the role of Swd2 WD40 domain in COMPASS-RNApII interactions, a point mutant was created at phenylalanine 250 , located at the center of the WD40 domain at the tip of propeller blade 6 (Supplementary Fig. 5a, b). Protein levels of 
a

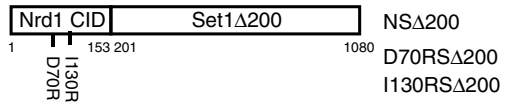

d

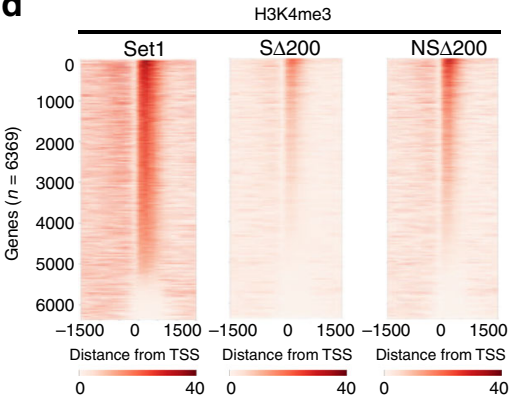

$\mathbf{f}$

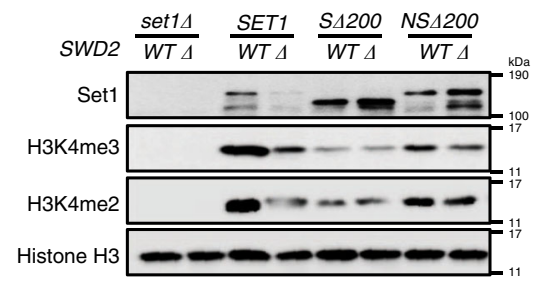

b

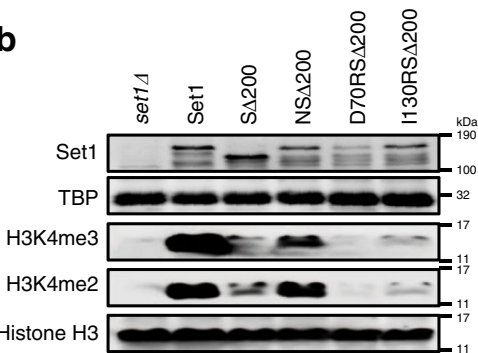

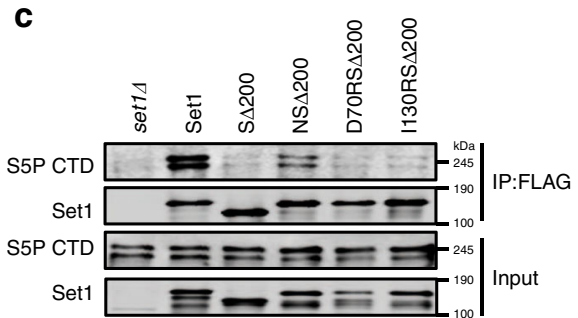

e

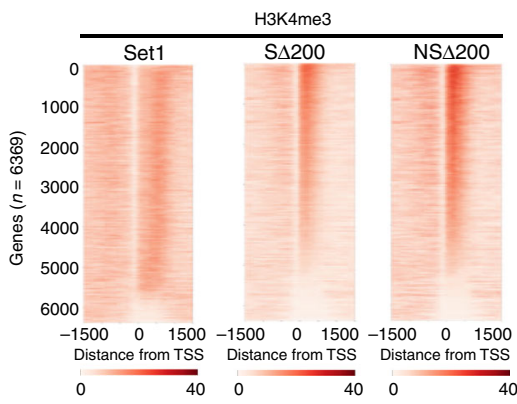

g

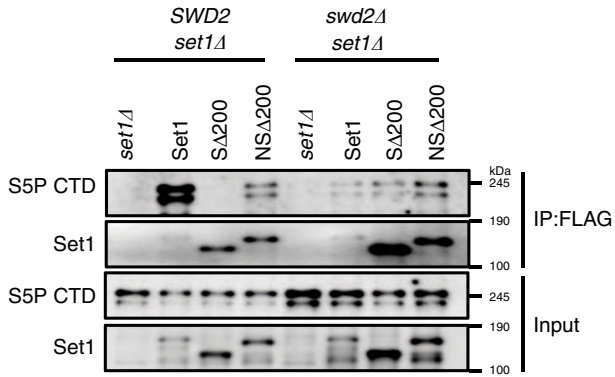

Fig. 4 Nrd1 CID fusion to Set1 200 partially restores CTD-binding and H3K4 methylation. a Schematic diagram of the NS $\Delta 200$ fusion protein consisting of the Nrd1 CID (amino acids 1-153) and Set1 200 . A FLAG-tag at the N-terminus is not shown. The positions of two CID mutants that disrupt CTD binding are also shown. b, c FLAG-tagged full-length Set1 (Set1), Set1 200 (S $\Delta 200$ ), or Nrd1 CID fusions (NS $\Delta 200$, D70RS $\Delta 200$, and I130RS $\Delta 200$ ) were transformed into set $1 \Delta$ cells. Whole-cell lysates (b) and proteins immunoprecipitated using anti-FLAG beads (c) were analyzed by immunoblotting using indicated antibodies. Cells transformed with empty plasmid (set14) served as a negative control. d, e ChIP-Seq heat maps of H3K4me3 (d) and H3K4me2 (e) from strains carrying full-length Set1 (Set1), Set1 200 (S $\Delta 200$ ), or Nrd1CID-Set1 200 fusion (NS $\Delta 200$ ). After normalizing to an internal spiked-in sample of S. pombe chromatin, SPMR values were mapped for individual RNApll transcriptional units and ordered according to H3K4me3 values in Set1 cells. H3K4me levels within 1500 bps from transcription start site (TSS) are shown. ChIP-seq reads from two technical repeats were averaged and

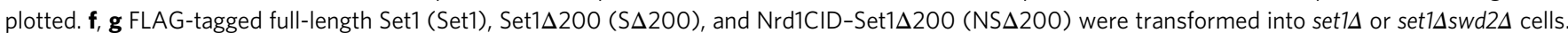
f Whole-cell lysates or $\mathbf{g}$ anti-FLAG immunoprecipitates were separated by SDS-PAGE and analyzed by immunoblotting using the indicated antibodies. Histone H3 was used as a loading control. Source data are provided as a Source data file.

the F250A mutant were close to those of wild-type Swd2. In contrast, H3K4me2 and me3 levels were much lower in the Swd2 mutant, indicating partial disruption of COMPASS activity (Fig. 5a). As we previously reported for other mutants with reduced H3K4 methylation, Set1 levels were also reduced (Fig. 5a). Immunoprecipitation of COMPASS via either Spp1 or Swd3, whose levels were unaffected in the mutant, or Swd2 itself, also showed that Swd2 F250A was defective for association with COMPASS (Supplementary Fig. 5c). To rule out that loss of interaction with Swd2 F250A was simply an indirect effect of Set1 degradation, we expressed HA-tagged WT or F250A Swd2 in the presence of FLAGtagged Set1 and untagged WT Swd2, thereby maintaining a supply of functional and stable COMPASS methylation activity. Immunoprecipitation of Set1 confirms that the F250A mutation strongly reduces Swd2 association with Set1 (Fig. 5b).

The yeast two-hybrid interaction between Set1 and the CTD was compared in cells containing WT or F250A Swd2. Similar to the Swd2 deletion (Fig. 3), the reduced interaction in the mutant background suggests that the Swd2 WD40 domain facilitates Set1/ COMPASS binding to RNApII (Fig. 5c). Immunoprecipitation with an antibody for CTD Ser5P pulled down less Spp1 and Swd2 in the F250A mutant, further supporting this conclusion (Supplementary Fig. 5d). Interestingly, ChIP-qPCR shows that Swd2 F250A is normally localized (Supplementary Fig. 5g), although this may reflect Swd2 in APT rather than free Swd2. However, Swd2 F250A diminishes enrichment of Spp1 and $\mathrm{H} 3 \mathrm{~K} 4 \mathrm{me} 3$ at the PMA1 5' region (Supplementary Fig. 5e, f), while Pol II occupancy is normal (Supplementary Fig. 5h). Supporting these in vivo results, reconstituted recombinant Set1/COMPASS complex incorporated lower levels of F250A than wild-type Swd2 (Fig. 5d) and exhibited reduced H3K4 methyltransferase activity on nucleosomes in vitro (Fig. 5e). Although these in vitro effects are less severe than in vivo, this is likely because H3K4 methylation defects in vivo are further amplified by Set1 degradation. Altogether, the F250A data implicate the Swd2 WD40 domain in COMPASS integrity and recruitment to early elongation complexes.

$\mathrm{H} 2 \mathrm{Bub}$ is required for $\mathrm{H} 3 \mathrm{~K} 4$ methylation independently of Swd2. Ubiquitylation of histone $\mathrm{H} 2 \mathrm{~B}$ on lysine 123 (H2Bub) 
a

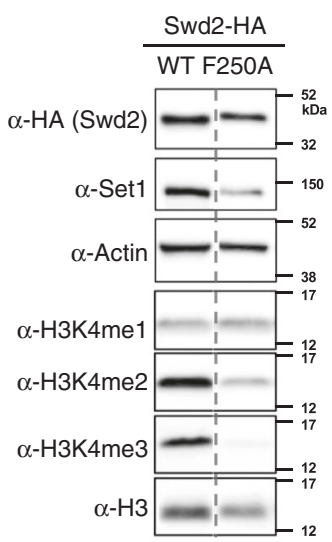

b

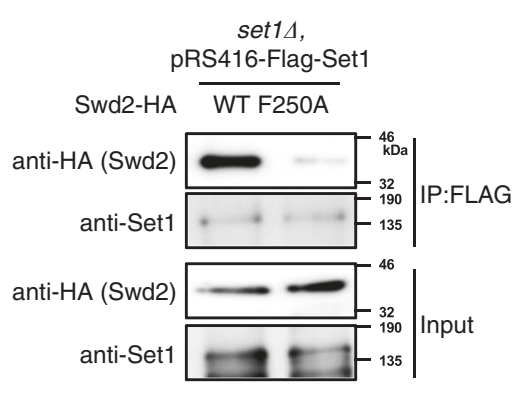

C

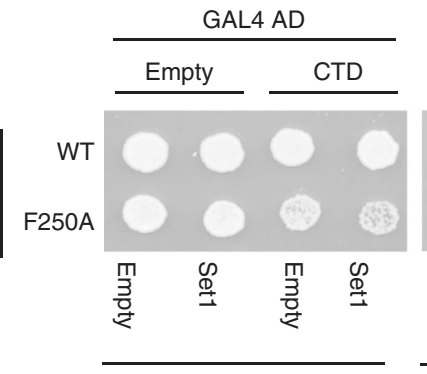

GAL4 AD
Ura, -Leu, -Trp

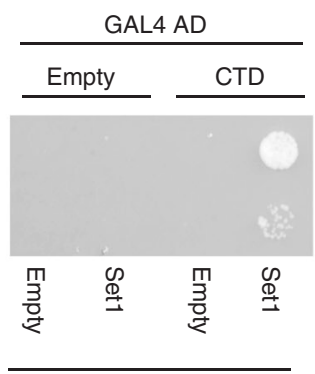

GAL4 AD

-Ura, -Leu, -Trp, -His

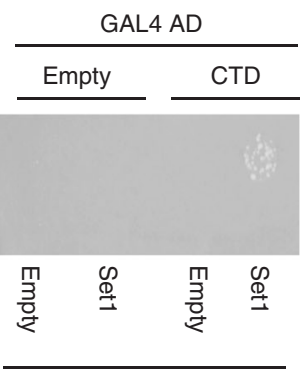

GAL4 AD

-Ura, -Leu, -Trp, -His

$5 \mathrm{mM} 3 \mathrm{AT}$ d

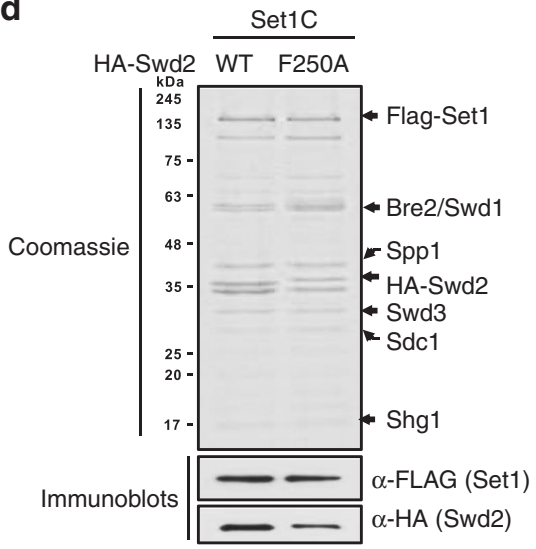

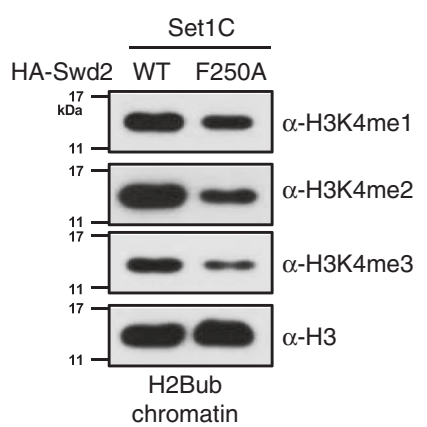

Fig. 5 The Swd2 WD40 domain is critical for COMPASS function. a Swd2-HA or Swd2-F250A-HA were expressed in an swd2 4 deletion strain. Protein levels were analyzed by immunoblotting using the indicated antibodies. Actin and Histone $\mathrm{H} 3$ were used as loading controls. Note that samples were run on the same blot, but intervening lanes were removed (dashed line, full blots appear in Source data). b Plasmid-encoded Swd2-HA and Swd2-F250A-HA were expressed in FLAG-Set1 containing cells that also express the endogenous untagged SWD2 gene in the chromosome. Proteins were immunoprecipitated with anti-FLAG beads (IP:FLAG) and then immunoblotted for Set1 and Swd2-HA. c The Y2H interaction between the Gal4BD-Set1 and Gal4AD-CTD fusions was tested in strain PJ69-4A expressing wild-type (WT) or mutant (F250A) Swd2. The empty Gal4 vectors served as negative controls. Activation of the Gal4-activated HIS3 reporter was tested on plates lacking histidine, with the addition of $5 \mathrm{mM} 3 \mathrm{AT}$ in the last panel for additional stringency. $\mathbf{d}$ Analysis of recombinant baculovirus-expressed Set1/COMPASS made with WT Swd2 or the Swd2-F250A mutant. SDS-PAGE/Coomassie blue staining shows total protein, while immunoblotting with anti-FLAG and anti-HA show Set1 and Swd2 levels, respectively. e H2Bub chromatin was subjected to in vitro histone methyltransferase assays ${ }^{16}$ with Swd2 WT and Swd2-F250A mutant-containing complexes shown in panel (d). H3K4 methylation status was monitored by immunoblotting with indicated antibodies. Histone H3 was used as an internal loading control. Source data are provided as a Source data file.

facilitates higher level H3K4 methylations by COMPASS 22 . $\mathrm{H} 2 \mathrm{Bub}$ is targeted to transcribed regions by the Paf1 elongation factor complex (Paf1C) 37,40 , which recruits the H2Bub ubiquitination complex Rad6-Bre1 ${ }^{20,21,41}$. Deleting any of these components causes loss of $\mathrm{H} 2 \mathrm{Bub}$ and reduced $\mathrm{H} 3 \mathrm{~K} 4 \mathrm{me} 2$ and me $3^{20,21,37}$. It has been proposed that Swd 2 mediates recognition of $\mathrm{H} 2 \mathrm{Bub}$ near $5^{\prime}$ ends of genes to facilitate binding of
COMPASS $^{22,23}$. If the primary role of $\mathrm{H} 2 \mathrm{Bub}$ was mediated via Swd2, the Swd2-independent fusion of Set1 to the Nrd1 CID might also bypass the requirement for H2Bub.

As expected, immunoblotting of cell extracts showed that H3K4me2 and me3 were reduced below the level of detection in paf1 $1 \Delta$ or rtfis cells (Fig. 6a), as well as in bre1s cells (Supplementary Fig. 6a). Replacing the first 200 amino acids of 
a
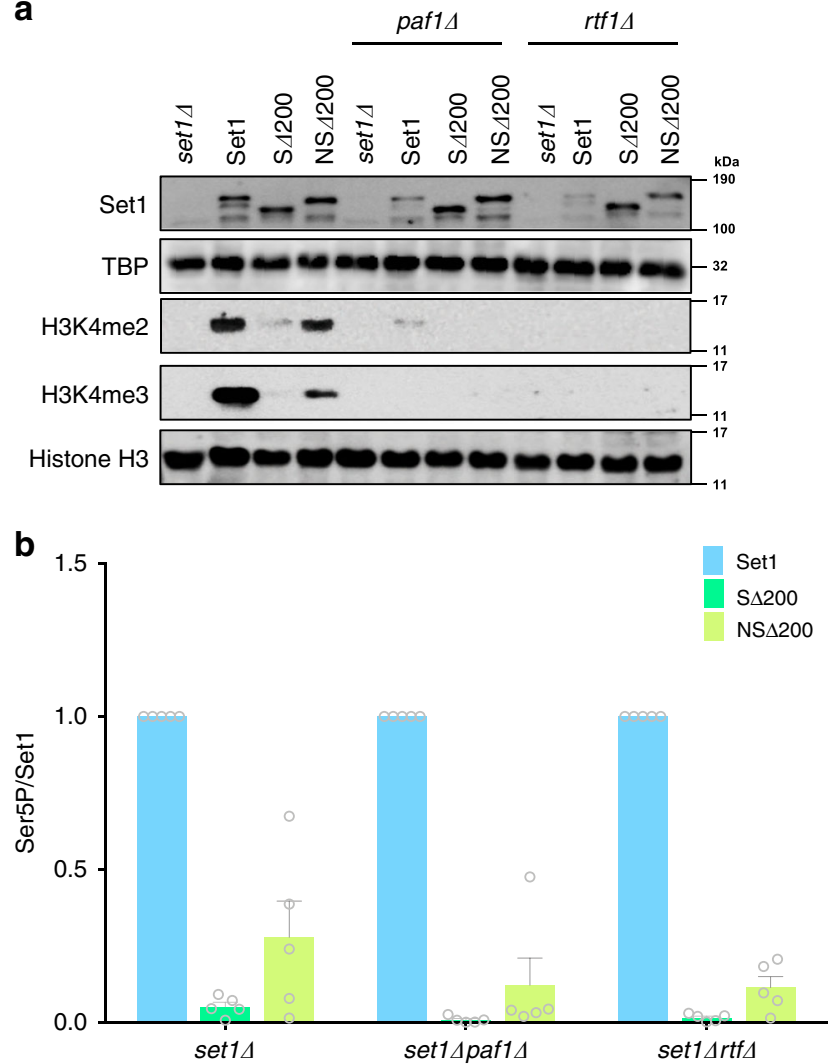

Fig. 6 Nrd1CID-Set1 200 fusion does not bypass the role of Paf1C and H2Bub in H3K4me. a Wild-type Set1 (Set1), Set1 200 (S $\Delta 200$ ), and Nrd1CID-Set1 200 (NS $\Delta 200$ ) were transformed into set14, set1 1 paf1 $1 \Delta$, or set $1 \Delta r t f 1 \Delta$ cells. Protein levels and histone methylations in whole-cell extracts were analyzed by immunoblotting. TBP and Histone $\mathrm{H} 3$ were used as loading controls. Parallel experiments in set1 $1 \mathrm{bre} 1 \Delta$ cells showed the same loss of H3K4 methylation (Supplemental Fig. 6a). b Set1 association with RNApll was quantitated by immunoprecipitating Set1 using $\alpha$-FLAG beads and probing for Rpb1 CTD Ser5P and Set1. Set1 and Ser5P signal intensities from each immunoblot were quantitated using ImageJ, and Ser5P signals were normalized to those for Set1. Circles show individual experimental values, and error bars show s.e.m. $(n=5)$. Values from wild type Set1 transformants were normalized to 1.0 for each experiment. Source data are provided as a Source data file.

Set1 with the Nrd1 CID partially restored the ability of Set1 to coimmunoprecipitate CTD-Ser5P, even in the Paf1C or Bre1 mutants (Fig. 6b, Supplementary Fig. 6b), but did not restore methylation (Fig. 6a, Supplementary Fig. 6a). The lack of methylation rescue was further confirmed by ChIP-qPCR on the YEF3 gene (Supplementary Fig. 7). These results echo our earlier demonstration that H3K4 methylation by a fusion of Set1 to the Rpb4 polymerase subunit is also still dependent upon $\mathrm{H} 2 \mathrm{Bub}^{35}$. Therefore, artificial recruitment of COMPASS does not bypass the requirement for $\mathrm{H} 2 \mathrm{Bub}$, indicating this modification promotes $\mathrm{H} 3 \mathrm{~K} 4$ methylation independently of COMPASS association with the RNApII elongation complex. This conclusion has been supported by several structures of nucleosome-bound COMPASS that were published while this paper was in review (see Discussion below) ${ }^{42-44}$.

\section{Discussion}

The details of how H3K4 methylation is coordinated with active transcription are still not completely understood. Although Set1/
COMPASS targeting is known to involve CTD Ser5 phosphorylation $^{34}$, it has been unclear whether COMPASS directly binds the CTD, whether RNApII domains other than CTD are involved, and which subunits of COMPASS mediate the interaction. Here, we present evidence that the N-terminal region of Set1 and Swd2 cooperatively interact with RNApII-CTD to promote proper recruitment of COMPASS to transcription elongation complexes at $5^{\prime}$ ends of genes. This model is based on the following observations:

i. Deleting the first 200 amino acids of Set1 (S $\Delta 200)$ strongly reduced $\mathrm{H} 3 \mathrm{~K} 4 \mathrm{me} 3$ and the ability to co-precipitate RNApII.

ii. Swd2, which interacts with amino acids 124-229 of Set $1^{16,19}$, is needed for strong CTD binding by Set 1 .

iii. Deleting residues 200-210 of Set1 abrogates Y2H interaction with both the Rpb1 CTD and Swd2.

iv. An Swd2 WD40 domain point mutation impairs its interaction with both Set1 and RNAPII, recruitment of COMPASS to chromatin, and $\mathrm{H} 3 \mathrm{~K} 4$ methylation activity.

v. Replacing amino acids 1-200 of Set1 with the Nrd1 CID (NS $\triangle 200)$ partially restores $\mathrm{H} 3 \mathrm{~K} 4 \mathrm{me}$ and Ser5P-CTD binding, showing that CTD binding is a major function of this region.

The Nrd1 CID fusion only partially substitutes for the Set1 Nterminal domain, which might reflect lower CTD affinity. More likely, Set1(1-200) and Swd2 have additional functions, beyond CTD interaction, in regulating COMPASS activity, as indicated by physical interaction studies ${ }^{16}$, protein crosslinking, and lowresolution cryo-EM structural analysis ${ }^{19}$. It appears that the Swd2-Set1 N-terminal module may fold over the catalytic body of COMPASS to contact other subunits and more C-terminal Set1 regions. An interesting speculative model is that CTD binding moves the Swd2-Set1 N-terminal module, relieving autoinhibition by making the Set1 active site more accessible.

Several facts led us to initially suspect that Swd2 would be the primary contact point with the CTD. Yeast Swd2 is also a component of the APT complex, which functions in the yeast Nrd1-Nab3-Sen1 (NNS) termination pathway for short nonpolyadenylated transcripts. Like COMPASS, NNS termination is also directed to early stage RNAPII elongation complexes marked by Ser5-phosphorylated $\mathrm{CTD}^{38}$, and this common CTD preference could be related to their shared subunit. Indeed, genetic evidence suggests APT and COMPASS compete for a common interacting protein, perhaps RNApII itself, through $S_{w d} 2^{26}$. Interestingly, $S$. pombe has two Swd2 homologs, each specific for one of the two complexes ${ }^{45}$. In addition, the mammalian Swd2 homolog Wdr82 helps recruit the Setd1A to chromatin and can directly bind to CTD Ser5P in vitro ${ }^{15}$. Echoing our results, this in vitro binding was stimulated by the $\mathrm{N}$-terminal region of mammalian Set1. Despite our expectations, we could not detect a CTD-Swd2 interaction in the $\mathrm{Y} 2 \mathrm{H}$ assay, nor did direct fusion of Swd2 to Set1 $\Delta 200$ rescue H3K4 methylation. Therefore, yeast Swd2 is apparently not sufficient for CTD interaction. Altogether, these results suggest that the N-terminal region of Set1 and Swd2 may form a composite binding site for tethering COMPASS to the RNApII CTD. It may be that both proteins make CTD contacts, or alternatively, that one triggers a conformation that renders the other competent for CTD binding.

In contrast to Set $1 \Delta 200$, Set1 derivatives further C-terminally truncated to amino acids $700^{35}$ or $762^{27,31}$ can tri-methylate $\mathrm{H} 3 \mathrm{~K} 4$. However, the resulting $\mathrm{H} 3 \mathrm{~K} 4 \mathrm{me} 3$ is mislocalized, spreading from the $5^{\prime}$ region into the gene bodies of active genes. This inaccurate distribution of $\mathrm{H} 3 \mathrm{~K} 4 \mathrm{me} 3$ still requires Spp1, as removal of the Spp1 interaction site by Set1 truncation to $780-1080$ leads to loss of $\mathrm{H} 3 \mathrm{~K} 4 \mathrm{me} 3^{18,27,31,46,47}$. Thus, other 
modes of COMPASS recruitment to chromatin must exist in the absence of the Set1 region 1-762, likely via direct interactions of the COMPASS catalytic domain with the nucleosome ${ }^{42-44}$, or perhaps through transient interactions between Swd2 and COMPASS subunits Spp1 and Swd1 ${ }^{19}$. In the context of fulllength Set1, these alternative modes of recruitment may be inhibited by the central autoinhibitory domain of Set $1^{27}$.

In addition to CTD binding, co-transcriptional methylation by COMPASS requires H2Bub. Paf1C associated with transcription elongation complexes directs Bre1/Rad6 ubiquitin ligase to nucleosomes, and $\mathrm{H} 2 \mathrm{Bub}$ in turn stimulates $\mathrm{H} 3 \mathrm{~K} 4$ methylation $36,37,48-50$. Although the Nrd1 CID fusion to Set1 can restore CTD binding, it does not bypass the requirement for H2Bub (Fig. 6, Supplemental Fig. 6). The H2Bub requirement also remained upon fusion of Set1 to RNApII subunit Rpb4 $4^{35}$ or strong overexpression of the hyperactive Set1 $\mathrm{N}$-terminal truncations ${ }^{30}$. After normalizing for reduced Set1 protein levels in the absence of $\mathrm{H} 3 \mathrm{~K} 4$ methylation ${ }^{30,35}$, the ability of Set1 to coprecipitate RNApII was also unaffected by loss of Paf1C or Rad6Bre1 ubiquitination complex (Fig. 6b). Therefore, H2Bub must act at a step after initial recruitment of COMPASS to the RNApII elongation complex. Indeed, several structures published while this paper was under review show how ubiquitin contacts and allosterically activates COMPASS ${ }^{42-44}$. While H2B-linked ubiquitin does not affect COMPASS affinity for nucleosomes ${ }^{14}$, its interactions with Set1 and other subunits rearrange the methyltransferase catalytic site into an active conformation.

A model thus emerges in which nucleosome binding, H2Bub sensing, and methyltransferase activity map to the C-terminal NSET/SET region of Set1 and associated COMPASS subunits, while an $\mathrm{N}$-terminal domain combines with Swd2 to create a CTDtargeting module. Future biochemical and structural studies will eventually reveal how these two modules interact, and how they are regulated by an intervening region of Set1 linked to autoinhibition and COMPASS degradation. The C-terminal N-SET/ SET domains and WRAD subunits are the most conserved among the Set1/MLL family, consistent with their common H3K4 methyltransferase activities. Our experiments suggest that the non-conserved $\mathrm{N}$-terminal domains are likely to target individual family members to different genomic locations through distinct protein interactions.

\section{Methods}

Strains, plasmids, and primers. Yeast strains used in this study are listed in Supplementary Table 1. Plasmids used for fusion protein analysis and yeast two hybrid assay are described in Supplementary Tables 2 and 3, respectively. DNA encoding the CID from Nrd1 (amino acids 1-153) was amplified from yeast genomic DNA and inserted into the indicated Setl constructs using isothermal assembly ${ }^{51}$. Primers used for cloning or ChIP assays are listed in Supplementary Table 4 .

Antibodies. The following antibodies were used in this study: anti-H3K4mel (Millipore Sigma 07-436, Burlington, MA, 1:2000), anti-H3K4me2 (Millipore Sigma 07-030, 1:1000), anti-H3K4me3 (Millipore Sigma 07-473 or 04-745, 1:2000), anti-H3 (Abcam 1791, Cambridge, UK, 1:3000), rat monoclonal anti-Ser2P CTD (3E10, Dirk Eick, 1:1000), rat monoclonal anti-Ser5P CTD (3E8, Dirk Eick, 1:3000), mouse monoclonal anti-CTD for total Rpb1 (8WG16, Buratowski lab, 1:1000), anti-Set1 (Santa Cruz, sc-101858, 1:1000), anti-TBP polyclonal antiserum (Buratowski lab, 1:3000), anti-Myc (MMS-150R-500, Covance, 1:2000), anti- $\beta$ Actin (Abcam 8224, 1:2000), anti-HA (3F10, Roche and 12CA5, 1:2000), anti-Gal4 DBD (sc-577, Santa Cruz, 1:1000)

Co-immunoprecipitation and immunoblotting. Whole-cell lysate was prepared from $100 \mathrm{ml}$ of yeast cultures grown at $30^{\circ} \mathrm{C}$ until $\mathrm{OD}_{600}$ reached to 1.0. Harvested cells were resuspended in $1 \mathrm{ml}$ lysis buffer (50 mM HEPES-KOH [pH 7.5], $150 \mathrm{mM}$ $\mathrm{NaCl}, 0.1 \%$ Triton X-100, 10\% Glycerol, $1 \mathrm{mM}$ DTT) supplemented with protease and phosphatase inhibitors $(1 \mu \mathrm{g} / \mathrm{ml}$ leupeptin, $1 \mu \mathrm{g} / \mathrm{ml}$ aprotinin, $1 \mu \mathrm{g} / \mathrm{ml}$ pepstatin $\mathrm{A}, 1 \mu \mathrm{g} / \mathrm{ml}$ antipain, $1 \mathrm{mM} \mathrm{NaF}, 1 \mathrm{mM} \mathrm{Na}_{3} \mathrm{VO}_{4}$, and $1 \mathrm{mM}$ PMSF). Protein concentrations were determined by Coomassie Protein Assay (Bio-Rad, Hercules, CA). For FLAG immunoprecipitation, $3 \mathrm{mg}$ of lysates were incubated at $4{ }^{\circ} \mathrm{C}$ overnight with $10 \mu \mathrm{l}$ of anti-DYKDDDDK L5 agarose beads (Biolegend, San Diego, CA) that had been preblocked with $0.1 \%$ bovine serum albumen. Beads were washed twice for $5 \mathrm{~min}$ with $1 \mathrm{ml}$ of lysis buffer.

To assay binding with purified proteins (Fig. $2 \mathrm{c}$ ), $5 \mu \mathrm{g}$ of purified yeast RNApII (generously provided by N. Adachi) and $100 \mathrm{ng}$ of Flag-tagged Setl complex purified from insect cells were incubated overnight at $4^{\circ} \mathrm{C}$ in $500 \mu \mathrm{l}$ of incubation buffer (50 mM HEPES, pH7.6, $100 \mathrm{mM}$ potassium acetate, $10 \mathrm{mM}$ magnesium acetate, $1 \mathrm{mM}$ EDTA, $10 \%$ glycerol and $0.1 \% \mathrm{NP}-40$ ). The mixtures were immunoprecipitated for $2 \mathrm{~h}$ at at $4{ }^{\circ} \mathrm{C}$ using $10 \mu \mathrm{l}$ of anti-DYKDDDDK L5 agarose beads that had been preblocked with $0.1 \%$ bovine serum albumen. Beads were washed three times for $5 \mathrm{~min}$ with $1 \mathrm{ml}$ of incubation buffer.

Immunoprecipitated proteins were eluted by boiling with $50 \mu \mathrm{l}$ of sodium dodecyl sulfate (SDS) sample buffer. For immunoblotting, 25-50 $\mu \mathrm{g}$ of whole cell lysates or $10 \mu \mathrm{l}$ of IP eluates were resolved by SDS polyacrylamide gel electrophoresis (PAGE) and transferred onto polyvinylidene fluoride membranes (Millipore, Billerica, MA). The membrane was blocked with blocking buffer ( $5 \%$ powdered skim milk in tris-buffered saline, $0.1 \%$ Tween-20) and probed with indicated antibodies above. Chemiluminescence signals were detected by using SuperSignal West Pico or Femto substrate (ThermoFisher Scientific, Waltham, MA) and visualized using the LAS 3000 image analyzer (Fuji Photo Film, Tokyo, Japan).

Chromatin Immunoprecipitation (ChIP). Chromatin samples were prepared as previously described using the following steps ${ }^{30}$. Cells were crosslinked with $1 \%$ formaldehyde for $20 \mathrm{~min}$, followed by $5 \mathrm{~min}$ quenching with $3 \mathrm{M}$ glycine. Cells were lysed by vortexing with glass beads $(30 \times 30 \mathrm{~s}$, with cooling between cycles, total 20) in FA lysis buffer (50 mM HEPES-KOH [pH7.5], $150 \mathrm{mM} \mathrm{NaCl}, 1 \mathrm{mM}$ EDTA, 1\% triton X-100, 0.1\% sodium deoxycholate, 0.5\% SDS, supplemented with protease inhibitors). Cell debris was removed by microcentrifugation, and the chromatin sheared to $\sim 200$ bp using a Misonix 3000 cup horn sonicator. Insoluble material was removed by microcentrifugation for $10 \mathrm{~min}$ at $14,000 \mathrm{rpm}$ at $4{ }^{\circ} \mathrm{C}$, and final protein concentration determined using Coomassie Protein Assay (Bio-Rad).

For immunoprecipitation, $500 \mu \mathrm{g}$ of chromatin was incubated with $0.5 \mu \mathrm{l}$ of anti-H3K4me2, or anti-H3K4me3 and $10 \mu \mathrm{l}$ of Protein G-Sepharose beads at $4{ }^{\circ} \mathrm{C}$ overnight in FA lysis buffer with SDS reduced to $0.1 \%$. For ChIP-Seq, chromatin from $S$. pombe was added at $10 \%$ relative to Saccharomyces cerevisiae chromatin as a "spike-in" control. Precipitates were washed with same buffer containing $275 \mathrm{mM}$ $\mathrm{NaCl}(\mathrm{H} 3 \mathrm{~K} 4 \mathrm{me} 2)$ or $500 \mathrm{mM} \mathrm{NaCl}(\mathrm{H} 3 \mathrm{~K} 4 \mathrm{me} 3)$. The beads were washed with Wash Buffer (10 mM Tris- $\mathrm{HCl}$ [pH 8.0], $0.25 \mathrm{M} \mathrm{LiCl,} 1 \mathrm{mM}$ EDTA, 0.5\% NP-40, $0.5 \%$ Na-Deoxycholate), and TE (10 mM Tris-HCl [pH8.0], 1 mM EDTA) buffer. Precipitated materials were eluted with buffer containing $50 \mathrm{mM}$ Tris- $\mathrm{HCl}$ [pH7.5], $10 \mathrm{mM}$ EDTA and $1 \%$ SDS by incubating at $65^{\circ} \mathrm{C}$ for $20 \mathrm{~min}$. Subsequent decrosslinking was performed at $42^{\circ} \mathrm{C}$ for $2 \mathrm{~h}$ and at $65^{\circ} \mathrm{C}$ for overnight with 0.8 $\mathrm{mg} / \mathrm{ml}$ of pronase (VWR, Radnor, PA). DNAs were phenol-chloroform extracted followed by ethanol-precipitation for further analysis.

Preparation of ChIP-Seq Libraries. Sequencing libraries were prepared using the following procedure, as previously described ${ }^{35}$. The concentration of immunoprecipitated DNA was measured using Qubit dsDNA HS Assay kit (Thermo Fisher Scientific). Barcoded sequencing libraries were generated from $1 \mathrm{ng}$ of immunoprecipitated DNA ${ }^{52}$. Briefly, immunoprecipitated DNA was end repaired using T4 DNA polymerase, T4 PNK, and DNA polymerase I Large (Klenow) fragment (New England Biolabs, Ipswich, MA). A single adenosine was added to the $3^{\prime}$ end of fragments using Klenow ( $3^{\prime}$ to $5^{\prime}$ exo minus, New England Biolabs) and then adapters containing multiplexing barcodes were ligated using T4 Quick DNA ligase (New England Biolabs). Adapter ligated DNA fragments between 200 and 500 bp were gel purified and PCR amplified with 16 cycles using Phusion DNA polymerase (ThermoFisher Scientific). Quality of libraries was examined using Agilent 2100 Bioanalyzer (Agilent Santa Clara, CA). Equimolar amount of each library was mixed and $50 \mathrm{bp}$ single-end sequenced in High Output (Standard) v3 Illumina HiSeq 2000 (Harvard Bauer Center Core Facility, Cambridge, MA).

Bioinformatics analysis. Analysis of ChIP-seq data was performed using the pipeline described in Soares et al. ${ }^{35}$. Sequence reads were demultiplexed using SABRE (https://github.com/najoshi/sabre.git) allowing for one mismatch in the barcode. For $S$. pombe spike-in normalization, demultiplexed reads were first aligned to the $S$. pombe genome (version ASM294v2.31). The unaligned reads were subsequently aligned to the $S$. cerevisiae genome (version R64-1-1). Only sequence reads that could be exclusively assigned to each genome were considered for total number of reads. Normalization factors were calculated by first calculating the proportion of reads of $S$. pombe versus total reads in input reads, and dividing the value by the square root (to account for the sequence tags per million reads (SPMR) normalization) of the proportion of reads of $S$. pombe vs. total reads in each immunoprecipitation reads. All alignment was performed using BOWTIE 1.1.1 $1^{53}$ excluding the first base and multi-aligned reads.

Conversion of alignment files was performed using SAMTOOLS $1.2^{54}$. Using MACS2.1.0 $0^{55}$ function, pileup tracks were calculated and duplicate reads were removed. Subsequently, tags were extended to 150 , and values were normalized to SPMR. Final coverage outputs were converted to high density wig files and 
analyzed using custom Python3.4 scripts (http://www.sciencelint.org/, https:// github.com/LuisSoares/Manuscript). The GEO accession number for the sequence data reported in this paper is GSE138281.

Yeast two-hybrid assay (Y2H). The SET1 open reading frame was cloned as a bait into vector pB66 (N-GAL4 BD-bait-C fusion). Initial interaction screening of a S.cerevisiae genomic library was performed by Hybrigenics, SA, Paris (https://www. hybrigenics-services.com/contents/our-services/interaction-discovery/ultimatey2h-2). For subsequent $\mathrm{Y} 2 \mathrm{H}$ validation and further analysis, S.cerevisiae reporter strains PJ69-4A or CG1945 were used. Gal4 DBD and Gal4 AD plasmids used in $\mathrm{Y} 2 \mathrm{H}$ are listed in Supplementary Table 3. Interactions were scored 2 or 3 days after spotting on SC media plates lacking the appropriate amino acids for reporter gene activation (Gal4-dependent UAS upstream of HIS3 or ADE2). For more stringent selection, 5 or $20 \mathrm{mM} 3$-aminotriazole (3-AT), a competitive inhibitor of the HIS3gene product, was added to media as indicated.

Quantitative PCR (qPCR) analysis. For individual gene analysis, quantitative PCR reactions of DNAs from ChIPs (see above) were done with a BioRad CFX384 using the following parameters: $5 \mathrm{~min}$ at $95^{\circ} \mathrm{C}, 40$ cycles of $15 \mathrm{~s}$ at $95^{\circ} \mathrm{C}, 15 \mathrm{~s}$ at $50^{\circ}$ $\mathrm{C}$, and $40 \mathrm{~s}$ at $72^{\circ} \mathrm{C}$, followed by $10 \mathrm{~min}$ at $95^{\circ} \mathrm{C}$. Oligonucleotides used for PCR reactions are listed in Supplementary Table 4.

COMPASS purification and histone methyltransferase assay. Purification of Set1 complex, H2Bub chromatin assembly, and in vitro methyltransferase assay were performed as described previously ${ }^{16,56}$. Briefly, cDNAs amplified from yeast genomic DNA were subcloned into pFASTBACl (ThermoFisher Scientific) with or without an epitope tag and baculoviruses were generated according to the manufacturer's instruction. Set 1 complexes containing Swd2 wild-type or F250A mutant were reconstituted from Sf9 cells which were infected with combinations of baculoviruses. Proteins/complexes were affinity purified using M2 agarose (Millipore Sigma, Burlington, MA). H2Bub chromatin assembly was performed using the recombinant ACF/NAP1 system. For recombinant chromatin methyltransferase assays, reactions containing $350 \mathrm{ng}$ (based on histone amount) recombinant chromatin ( $35 \mu \mathrm{l}$, assembled as above), purified Set1 complexes and $100 \mu \mathrm{M}$ SAM (S-adenosyl methionine, New England Biolabs) were adjusted to $40 \mu \mathrm{l}$ with HEG buffer (25 mM HEPES [pH 7.6], $0.1 \mathrm{mM}$ EDTA and $10 \%$ glycerol) and incubated at $30^{\circ} \mathrm{C}$ for $1 \mathrm{~h}$. Proteins were resolved by SDSPAGE and subjected to immunoblotting.

Statistics and reproducibility. All western blots were repeated at least three times and representative images were shown in this paper. Unpaired $t$ test was used for statistical analysis. For ChIP-Seq, mean values from two biological replicates are represented.

Reporting summary. Further information on research design is available in the Nature Research Reporting Summary linked to this article.

\section{Data availability}

The ChIP-seq data sets generated and analyzed in Fig. 4 and Suplementary Fig. 4 are available in the GEO repository, accession number GSE138281 (https://www.ncbi.nlm. nih.gov/geo/query/acc.cgi?acc=GSE138281). All other relevant data supporting the key findings of this study are available within the article and its Supplementary Information files or from the corresponding authors upon reasonable request. Uncropped images for gels and blots in all figures are provided in the Source data file. A reporting summary for this Article is available as a Supplementary Information file.

Received: 1 October 2019; Accepted: 14 April 2020;

Published online: 01 May 2020

\section{References}

1. Kouzarides, T. Chromatin modifications and their function. Cell 128, 693-705 (2007).

2. Li, B., Carey, M. \& Workman, J. L. The role of chromatin during transcription. Cell 128, 707-719 (2007).

3. Shilatifard, A. Chromatin modifications by methylation and ubiquitination: implications in the regulation of gene expression. Annu. Rev. Biochem. 75, 243-269 (2006).

4. Ernst, P. \& Vakoc, C. R. WRAD: enabler of the SET1-family of H3K4 methyltransferases. Brief. Funct. Genomics 11, 217-226 (2012).

5. Smith, E. \& Shilatifard, A. The chromatin signaling pathway: diverse mechanisms of recruitment of histone-modifying enzymes and varied biological outcomes. Mol. Cell 40, 689-701 (2010).
6. Briggs, S. D. et al. Histone H3 lysine 4 methylation is mediated by Set1 and required for cell growth and rDNA silencing in Saccharomyces cerevisiae. Genes Dev. 15, 3286-3295 (2001).

7. Roguev, A. et al. The Saccharomyces cerevisiae Set1 complex includes an Ash2 homologue and methylates histone 3 lysine 4. EMBO J. 20, 7137-7148 (2001).

8. Miller, T. et al. COMPASS: a complex of proteins associated with a trithoraxrelated SET domain protein. Proc. Natl Acad. Sci. USA 98, 12902-12907 (2001).

9. Nagy, P. L., Griesenbeck, J., Kornberg, R. D. \& Cleary, M. L. A trithorax-group complex purified from Saccharomyces cerevisiae is required for methylation of histone H3. Proc Natl Acad Sci USA 99, 90-94 (2002).

10. Dichtl, B., Aasland, R. \& Keller, W. Functions for S. cerevisiae Swd2p in 3' end formation of specific mRNAs and snoRNAs and global histone 3 lysine 4 methylation. RNA 10, 965-977 (2004).

11. Nedea, E. et al. Organization and function of APT, a subcomplex of the yeast cleavage and polyadenylation factor involved in the formation of mRNA and small nucleolar RNA 3'-ends. J. Biol. Chem. 278, 33000-33010 (2003).

12. Dehe, P. M. et al. Protein interactions within the Set 1 complex and their roles in the regulation of histone 3 lysine 4 methylation. J. Biol. Chem. 281, 35404-35412 (2006)

13. Halbach, A. et al. Cotranslational assembly of the yeast SET1C histone methyltransferase complex. EMBO J. 28, 2959-2970 (2009).

14. Kim, J. et al. The n-SET domain of Set1 regulates H2B ubiquitylationdependent H3K4 methylation. Mol. Cell 49, 1121-1133 (2013).

15. Lee, J. H. \& Skalnik, D. G. Wdr82 is a C-terminal domain-binding protein that recruits the Setd1A Histone H3-Lys4 methyltransferase complex to transcription start sites of transcribed human genes. Mol. Cell Biol. 28 609-618 (2008).

16. Jeon, J., McGinty, R. K., Muir, T. W., Kim, J. A. \& Kim, J. Crosstalk among Set1 complex subunits involved in H2B ubiquitylation-dependent H3K4 methylation. Nucleic acids Res. 46, 11129-11143 (2018).

17. Hsu, P. L. et al. Crystal structure of the COMPASS H3K4 methyltransferase catalytic module. Cell 174(1106-1116), e1109, https://doi.org/10.1016/j. cell.2018.06.038 (2018).

18. Qu, Q. et al. Structure and conformational dynamics of a COMPASS histone H3K4 methyltransferase complex. Cell 174(1117-1126), e1112 (2018).

19. Wang, Y. et al. Architecture and subunit arrangement of the complete Saccharomyces cerevisiae COMPASS complex. Sci. Rep. 8, 17405 (2018).

20. Dover, J. et al. Methylation of histone H3 by COMPASS requires ubiquitination of histone H2B by Rad6. J. Biol. Chem. 277, 28368-28371 (2002).

21. Sun, Z. W. \& Allis, C. D. Ubiquitination of histone H2B regulates H3 methylation and gene silencing in yeast. Nature 418, 104-108 (2002).

22. Soares, L. M. \& Buratowski, S. Histone crosstalk: H2Bub and H3K4 Methylation. Mol. Cell 49, 1019-1020 (2013).

23. Lee, J. S. et al. Histone crosstalk between $\mathrm{H} 2 \mathrm{~B}$ monoubiquitination and $\mathrm{H} 3$ methylation mediated by COMPASS. Cell 131, 1084-1096 (2007).

24. Vitaliano-Prunier, A. et al. Ubiquitylation of the COMPASS component Swd2 links H2B ubiquitylation to H3K4 trimethylation. Nat. Cell Biol. 10, 1365-1371 (2008)

25. Nedea, E. et al. The Glc7 phosphatase subunit of the cleavage and polyadenylation factor is essential for transcription termination on snoRNA genes. Mol. cell 29, 577-587 (2008).

26. Soares, L. M. \& Buratowski, S. Yeast Swd2 is essential because of antagonism between Set1 histone methyltransferase complex and APT (associated with Pta1) termination factor. J. Biol. Chem. 287, 15219-15231 (2012).

27. Schlichter, A. \& Cairns, B. R. Histone trimethylation by Set1 is coordinated by the RRM, autoinhibitory, and catalytic domains. EMBO J. 24, 1222-1231 (2005).

28. Tresaugues, L. et al. Structural characterization of Set1 RNA recognition motifs and their role in histone H3 lysine 4 methylation. J. Mol. Biol. 359 , 1170-1181 (2006)

29. Luciano, P. et al. Binding to RNA regulates Set1 function. Cell Discov. 3, 17040 (2017).

30. Soares, L. M., Radman-Livaja, M., Lin, S. G., Rando, O. J. \& Buratowski, S. Feedback control of Set1 protein levels is important for proper H3K4 methylation patterns. Cell Rep. 6, 961-972 (2014).

31. Thornton, J. L. et al. Context dependency of Set1/COMPASS-mediated histone H3 Lys4 trimethylation. Genes Dev. 28, 115-120 (2014).

32. Corden, J. L. RNA polymerase II C-terminal domain: tethering transcription to transcript and template. Chem. Rev. 113, 8423-8455 (2013).

33. Buratowski, S. Progression through the RNA polymerase II CTD cycle. Mol. Cell 36, 541-546 (2009).

34. Ng, H. H., Robert, F., Young, R. A. \& Struhl, K. Targeted recruitment of Set1 histone methylase by elongating Pol II provides a localized mark and memory of recent transcriptional activity. Mol. Cell 11, 709-719 (2003).

35. Soares, L. M. et al. Determinants of histone H3K4 methylation patterns. Mol. Cell 68(773-785), e776 (2017)

36. Wood, A., Schneider, J., Dover, J., Johnston, M. \& Shilatifard, A. The Paf1 complex is essential for histone monoubiquitination by the Rad6-Bre1 
complex, which signals for histone methylation by COMPASS and Dotlp. J. Biol. Chem. 278, 34739-34742 (2003).

37. Krogan, N. J. et al. The Paf1 complex is required for histone H3 methylation by COMPASS and Dot1p: linking transcriptional elongation to histone methylation. Mol. Cell 11, 721-729 (2003).

38. Vasiljeva, L., Kim, M., Mutschler, H., Buratowski, S. \& Meinhart, A. The Nrd1Nab3-Sen1 termination complex interacts with the Ser5-phosphorylated RNA polymerase II C-terminal domain. Nat. Struct. Mol. Biol. 15, 795-804 (2008).

39. Homann, O. R. \& Johnson, A. D. MochiView: versatile software for genome browsing and DNA motif analysis. BMC Biol. 8, 49 (2010).

40. Squazzo, S. L. et al. The Pafl complex physically and functionally associates with transcription elongation factors in vivo. EMBO J. 21, 1764-1774 (2002).

41. Van Oss, S. B., Cucinotta, C. E. \& Arndt, K. M. Emerging Insights into the Roles of the Paf1 Complex in Gene Regulation. Trends Biochem. Sci. 42, 788-798 (2017).

42. Hsu, P. L. et al. Structural basis of H2B ubiquitination-dependent H3K4 methylation by COMPASS. Mol. Cell 76(712-723), e714 (2019).

43. Worden, E. J., Zhang, X. \& Wolberger, C. Structural basis for COMPASS recognition of an H2B-ubiquitinated nucleosome. eLife https://doi.org/ 10.7554/eLife.53199 (2020).

44. Xue, H. et al. Structural basis of nucleosome recognition and modification by MLL methyltransferases. Nature 573, 445-449 (2019).

45. Roguev, A. et al. A comparative analysis of an orthologous proteomic environment in the yeasts Saccharomyces cerevisiae and Schizosaccharomyces pombe. Mol. Cell. Proteom. 3, 125-132 (2004).

46. Fingerman, I. M., Wu, C. L., Wilson, B. D. \& Briggs, S. D. Global loss of Set1mediated $\mathrm{H} 3$ Lys4 trimethylation is associated with silencing defects in Saccharomyces cerevisiae. J. Biol. Chem. 280, 28761-28765 (2005).

47. Acquaviva, L. et al. The COMPASS subunit Spp1 links histone methylation to initiation of meiotic recombination. Science 339, 215-218 (2013).

48. Ng, H. H., Dole, S. \& Struhl, K. The Rtfl component of the Pafl transcriptional elongation complex is required for ubiquitination of histone H2B. J. Biol. Chem. 278, 33625-33628 (2003).

49. Battaglia, S. et al. RNA-dependent chromatin association of transcription elongation factors and Pol II CTD kinases. eLife https://doi.org/10.7554/ eLife.25637 (2017).

50. Kim, J. et al. RAD6-Mediated transcription-coupled H2B ubiquitylation directly stimulates H3K4 methylation in human cells. Cell 137, 459-471 (2009).

51. Gibson, D. G. et al. Enzymatic assembly of DNA molecules up to several hundred kilobases. Nat. Methods 6, 343-345 (2009).

52. Wong, K. H., Jin, Y. \& Moqtaderi, Z. Multiplex Illumina sequencing using DNA barcoding. Curr. Protoc. Mol. Biol. https://doi.org/10.1002/0471142727. mb0711s101 (2013).

53. Langmead, B., Trapnell, C., Pop, M. \& Salzberg, S. L. Ultrafast and memoryefficient alignment of short DNA sequences to the human genome. Genome Biol. 10, R25 (2009).

54. Li, H. et al. The sequence Alignment/Map format and SAMtools. Bioinformatics 25, 2078-2079 (2009).

55. Feng, J., Liu, T., Qin, B., Zhang, Y. \& Liu, X. S. Identifying ChIP-seq enrichment using MACS. Nat. Protoc. 7, 1728-1740 (2012).

56. Kim, J. \& Roeder, R. G. Nucleosomal H2B ubiquitylation with purified factors. Methods 54, 331-338 (2011).

\section{Acknowledgements}

H.B. was supported by a postdoctoral fellowship from the Basic Science Research Program of the National Research Foundation of Korea (NRF), funded by the Ministry of Education (2015R1A6A3A03017730). This work was supported by grants from the National Research Foundation of Korea (NRF-2019R1A2C2090830) to J.K., from "Ligue Nationale Contre le Cancer" (LNCC) (Equipe labellisée) to M.D. and V.G., and grants GM046498 and GM056663 from the U.S. National Institutes of Health to S.B. We thank D. Eick (CIPS, Munich) for CTD antibodies, S. Briggs (Purdue) for the parent FLAG-Set1 construct, C. Gwizdek (Dargemont lab) for creating the Swd2 F250A mutant, and N. Adachi (SBRC, KEK, Japan) for purified RNApII.

\section{Author contributions}

H.B. and M.D. performed all the experiments, with the exception of COMPASS reconstitution experiments performed by J.J. and J.K., and computational analyses of genomic data by L.S. and H.B. C.D. identified and initially characterized the SWD2 F250A mutant. H.B., M.D., V.G., and S.B. participated in experimental conception and design, and wrote the paper with input from all other authors.

\section{Competing interests}

The authors declare no competing interests.

\section{Additional information}

Supplementary information is available for this paper at https://doi.org/10.1038/s41467020-16082-2.

Correspondence and requests for materials should be addressed to V.G. or S.B.

Peer review information Nature Communications thanks Yali Dou and the other, anonymous, reviewer(s) for their contribution to the peer review of this work. Peer reviewer reports are available.

Reprints and permission information is available at http://www.nature.com/reprints

Publisher's note Springer Nature remains neutral with regard to jurisdictional claims in published maps and institutional affiliations.

(c) (i)

Open Access This article is licensed under a Creative Commons Attribution 4.0 International License, which permits use, sharing, adaptation, distribution and reproduction in any medium or format, as long as you give appropriate credit to the original author(s) and the source, provide a link to the Creative Commons license, and indicate if changes were made. The images or other third party material in this article are included in the article's Creative Commons license, unless indicated otherwise in a credit line to the material. If material is not included in the article's Creative Commons license and your intended use is not permitted by statutory regulation or exceeds the permitted use, you will need to obtain permission directly from the copyright holder. To view a copy of this license, visit http://creativecommons.org/ licenses/by/4.0/.

(C) The Author(s) 2020 